\title{
EBIC contrast theory of dislocations : intrinsic recombination properties
}

\author{
J. L. Farvacque and B. Sieber \\ Laboratoire de Structure et Propriétés de l'Etat Solide, URA 234, Bâtiment C6, Université des Sciences et \\ Techniques de Lille Flandres-Artois, 59655 Villeneuve d'Ascq Cedex, France
}

(Reçu le 31 juillet 1989, révisé le 22 décembre 1989, accepté le 5 janvier 1990)

\begin{abstract}
Résumé. - Nous proposons un modèle théorique du contraste EBIC de dislocations perpendiculaires à la surface. Nous traitons le cas de la zone de diffusion des barrières de Schottky perpendiculaires au faisceau d'électrons. Le faisceau est statique et à l'aplomb de la ligne de dislocation. Les mécanismes de recombinaison à la dislocation sont associés de façon self-consistente à l'existence d'un champ électrique permanent dont l'effet est simplement décrit en entourant la ligne de dislocation d'un volume à l'intérieur duquel il n'y a pas diffusion des porteurs libres. La surface limitant ce volume est donc affectée d'une vitesse de recombinaison infinie. Ces nouvelles conditions aux limites conduisent à une expression du contraste EBIC constituée de deux termes. Le premier correspond à l'expression usuelle du contraste établi dans les théories précédentes; le second provient de la modification importante apportée par la dislocation à la distribution des porteurs minoritaires, ne justifiant plus l'utilisation de l'approximation de Born. Ce dernier terme joue un rôle essentiel, dans le sens qu'il est la plupart du temps plus important dans la formation du contraste que le premier. Les contrastes EBIC ainsi calculés numériquement sont de l'ordre de quelques pourcents, ce qui est tout à fait l'ordre de grandeur de ce qui est habituellement observé.
\end{abstract}

\begin{abstract}
A physical model of the electron-beam-induced contrast (EBIC) of dislocations perpendicular to the surface is proposed. The theory deals with the diffusion region of Schottky barriers perpendicular to the electron beam. As the geometry investigated is such that the electron beam impinges directly above the dislocation line, the maximum value of the contrast is calculated. The carrier recombination at the dislocation is introduced in the diffusion equation with the help of its self-consistent electric field which is permanent in a volume $V_{\mathrm{D}}$ around the dislocation line; therefore, we make the assumption that no diffusion of free carriers can occur inside $V_{\mathrm{D}}$ and an infinite recombinaison rate is assigned to the surface limiting $V_{\mathrm{D}}$. The resulting expression for the contrast is made of two terms ; the first corresponds to the usual expression for the contrast given in previous models; the second originates in the modification of the carrier density brought by the dislocation. It is shown that the latter contribution to the contrast is generally greater than the first one. Numerical results indicate that intrinsic recombination at dislocations can lead to EBIC contrasts of a few percents as observed experimentally.
\end{abstract}

\section{Introduction.}

Over the last 10 years, the EBIC contrast of dislocations in the bulk region of Schottky diodes or pn junctions has been the subject of various theoretical calculations which can be classified in two main groups ; in the first, the dislocation is represented by a cylinder within which the minority carrier lifetime is reduced compared with that in the bulk [1-5]. A defect strength is thus associated with the dislocation, and the theoretical problem is to solve the diffusion equation in this inhomogeneous region subject to various approximations [1-5]. Reliable solutions of the diffusion equation have been proposed, but the defect strength has no direct physical meaning and is an adjustable phenomenological parameter. The second class of calculation does not solve the diffusion equation, but introduces the dislocation electronic properties via the spatial extension of its electric field $[6,7]$. Thus, neither approach allows one to evaluate from first principles the correct order of magnitude of the EBIC contrast, yielding only its qualitative variation versus the accelerating voltage [2], temperature [6-10] or beam intensity [7]. In this paper we present a physical approach to the calculation of the EBIC contrast, 
which takes into account the diffusion of minority carriers as well as the physical properties of the dislocation. Here, the simplest geometry of a straight dislocation perpendicular to the Schottky diode will be considered. Our calculation leads to a simple expression for the maximum contrast value of the contrast of dislocations for any type of generation function, but the mathematical treatment prevents us (for the moment) to take rigorously into account the eventual effect of saturation.

\section{EBIC contrast of dislocations.}

The EBIC contrast of a dislocation is expressed as $c=\delta I / I_{0}$, where $I_{0}$ is the background current taken far from any defect, and $\delta I$ is the variation of the collected current due to the defect recombination mechanism. In the following, we will deal with a Schottky diode parallel to the surface, which is one of the best configurations to study the recombination properties of defects in bulk semiconductors. Current collection and defect recombination in the space charge region are not taken into account in the present calculation.

We will first recall the fundamental equations describing the diffusion of minority carriers without any defect, as well as the associated Green function ; they will all be useful in the determination of the contrast.

The surface $\Sigma$ of the diode, located at $z=0$, is the collecting plane for the excess minority carriers created by the incident electron beam in the bulk. The background current $I_{0}$ is the flux of the excess minority carriers current density $\mathbf{j}_{0}$ in the bulk region through $\Sigma$ :

$$
I_{0}=-\left.\int_{\Sigma} \mathbf{j}_{0}\right|_{z=0} \mathrm{~d} \mathbf{S}
$$

$\mathbf{j}_{0}$ satisfies the continuity equation which is expressed, under steady state and low injection level conditions in an n-type semiconductor, by:

$$
\operatorname{div} \mathbf{j}_{0}=e \cdot g(\mathbf{r})-\frac{e \cdot p_{0}(\mathbf{r})}{\tau}
$$

$p_{0}(\mathbf{r})$ is the minority carrier density far from any defect ; $\tau$ and $g(\mathbf{r})$ are the minority carrier lifetime and generation function respectively. In isothermal conditions, $\mathbf{j}_{0}$ is made of a drift and of a diffusion contribution :

$$
\mathbf{j}_{0}=\sigma \mathbf{E}-e D \operatorname{grad} p_{0}(\mathbf{r}) .
$$

Without any electric field, the diffusion component is the only one which plays a role in the bulk region, so that :

$$
\Delta p_{0}(\mathbf{r})=\frac{p_{0}(\mathbf{r})}{L^{2}}-g(\mathbf{r}) / D
$$

with $L=(D \tau)^{1 / 2}$ the bulk minority carrier diffusion length. The latter equation can be solved for any generation function $g(\mathbf{r})$ by use of the Green's function formalism [1, 11]. The integral form of $p_{0}(\mathbf{r})$ is simply given by

$$
p_{0}(\mathbf{r})=\int_{V_{\text {crystal }}} \chi\left(\mathbf{r}, \mathbf{r}^{\prime}\right) \frac{g\left(\mathbf{r}^{\prime}\right)}{D} \mathrm{~d} \mathbf{r}^{\prime}
$$

where $V_{\text {crystal }}$ is the semi infinite volume of the crystal bounded by the collecting $\Sigma$ surface, and the Green function $\chi\left(\mathbf{r}, \mathbf{r}^{\prime}\right)$ is the standard solution of :

$$
\Delta \chi\left(\mathbf{r}, \mathbf{r}^{\prime}\right)=\frac{\chi\left(\mathbf{r}, \mathbf{r}^{\prime}\right)}{L^{2}}-\delta\left(\mathbf{r}-\mathbf{r}^{\prime}\right)
$$

for a semi infinite medium. The boundary conditions for $\chi\left(\mathbf{r}, \mathbf{r}^{\prime}\right)$ are the same as those for $p_{0}(\mathbf{r})$ which is zero at the $\Sigma$ surface. Thus, $\chi$ can be found by the method of images and is given by [1] :

$$
\begin{aligned}
\chi\left(\mathbf{r}, \mathbf{r}^{\prime}\right)=\frac{1}{4 \pi}\left[\frac{\exp \left(-\left|\mathbf{r}-\mathbf{r}^{\prime}\right| / L\right)}{\left|\mathbf{r}-\mathbf{r}^{\prime}\right|}-\right. \\
\left.-\frac{\exp \left(-\left|\mathbf{r}-\mathbf{r}^{\prime \prime}\right| / L\right)}{\left|\mathbf{r}-\mathbf{r}^{\prime \prime}\right|}\right]
\end{aligned}
$$

where $\mathbf{r}^{\prime}$ and $\mathbf{r}^{\prime \prime}$ are respectively the coordinates of the point source and its image with respect to $\Sigma$ surface.

Using (1), introducing $\mathbf{j}_{0}=-e D$ grad $\left[p_{0}(\mathbf{r})\right]$ and the $z$ derivative of (7), one obtains :

$$
I_{0}=e \int_{V_{\text {crystal }}} \mathrm{e}^{-z / L} g(\mathbf{r}) \mathrm{d} \mathbf{r} .
$$

We will consider the role of intrinsic recombination at the dislocation on the EBIC contrast i.e. the case where the dislocation acts as a recombination centre due to its own electronic levels. These levels are here depicted as a one dimensional electronic band introduced in the forbidden band gap. In such situations, the electrostatic interaction energy of the charges trapped at the dislocation results in local electric fields, which are repulsive for majority carriers.

Among the minority carriers created close to a dislocation, those which reach the dislocation by diffusion, or those created within the electric field area, are instantaneously attracted by the local fields towards the dislocation line (similar to what happens within the depleted region of a Schottky diode). The equilibrium charge of the dislocation is therefore decreased unless the carriers recombine at the dislocation. If the minority carrier recombination mechanisms at the dislocation line are efficient enough to eliminate the trapped minority carriers, the dislocation electric fields remain unchanged. If not, the dislocation charge reaches another equilibrium which reduces the spatial extension of the 
electric field and thus the minority carrier collection (saturation effect). In any case a steady state electric field is established and recombination at dislocation can be simply depicted by introducing in the diffusion equation a permanent and selfconsistent dislocation electric field :

$$
D \Delta p(\mathbf{r})=\frac{p(\mathbf{r})}{\tau}-g(\mathbf{r})+\frac{1}{e} \operatorname{div}(\sigma \mathbf{E}(\mathbf{r})) .
$$

Solutions of equation (9) can also been expressed with help of the previous Green function $\chi\left(\mathbf{r}, \mathbf{r}^{\prime}\right)$; but now, the term $g(\mathbf{r}) / D-\operatorname{div}(\sigma \mathbf{E}) / e D$ has to be substituted for $g(\mathbf{r}) / D$. This gives :

$$
\begin{aligned}
p(\mathbf{r})=\frac{1}{D} \int_{V_{\text {crystal }}} \chi\left(\mathbf{r}, \mathbf{r}^{\prime}\right) \times \\
\times\left[g\left(\mathbf{r}^{\prime}\right)-\frac{1}{e} \operatorname{div}\left\{\sigma \mathbf{E}\left(\mathbf{r}^{\prime}\right)\right\}\right] \mathrm{d} \mathbf{r}^{\prime} .
\end{aligned}
$$

The current $I$ collected at the Schottky diode in presence of a dislocation is obtained following the previous procedure ; it is easy to see that the current variation $\delta I$ is given by :

$$
\delta I=-\int_{V_{\text {crystal }}} \mathrm{e}^{-z / L} \operatorname{div}[\sigma \mathbf{E}(\mathbf{r})] \mathrm{dr} .
$$

The dislocation electrical field is a continuous function whose amplitude is decreasing with the distance to the dislocation line. However, let us make the assumption that it is indeed located within a volume $V_{\mathrm{d}}$ surrounding the dislocation line. Thus, integral (11) can be written as :

$$
\delta I=-\int_{V_{\mathrm{d}}} \mathrm{e}^{-z / L} \operatorname{div}[\boldsymbol{\sigma E}(\mathbf{r})] \mathrm{d} \mathbf{r}
$$

since the electrical field is zero outside $V_{\mathrm{d}}$.

The carrier density in presence of the dislocation can be expressed as :

$$
p(\mathbf{r})=p_{0}(\mathbf{r})+\delta p(\mathbf{r})
$$

where $p_{0}(\mathbf{r})$ is solution of the diffusion equation without defect; we can therefore replace div $[\sigma \mathbf{E}]$ given by (9) by its following equivalent expression :

$$
\operatorname{div}[\sigma \mathrm{E}]=e\left\{\Delta \delta p(\mathbf{r})-\frac{p(\mathbf{r})}{\tau}+\frac{p_{0}(\mathbf{r})}{\tau}\right\}
$$

this gives for $\delta I$ :

$$
\begin{aligned}
\delta I= & -\frac{e}{\tau} \int_{V_{\mathrm{d}}} \mathrm{e}^{-z / L}\left\{p_{0}(\mathbf{r})-p(\mathbf{r})\right\} \mathrm{d} \mathbf{r} \\
& +\int_{V_{\mathrm{d}}} \mathrm{e}^{-z / L} \operatorname{div}[\operatorname{grad} \delta p(\mathbf{r})] \mathrm{d} \mathbf{r} .
\end{aligned}
$$

Using the identity :

$$
\begin{aligned}
& e^{-z / L} \operatorname{div}[\operatorname{grad} \delta p(\mathbf{r})]= \\
&= \operatorname{div}\left[\mathrm{e}^{-z / L} \operatorname{grad} \delta p(\mathbf{r})\right] \\
&-\operatorname{grad} \delta p(\mathbf{r}) \cdot \operatorname{grad}\left(\mathrm{e}^{-z / L}\right) .
\end{aligned}
$$

Since the total electrical current is conservative (a pure diffusion current outside $V_{d}$ and a pure drift one inside), Ostrogradsky's theorem can be used in order to transform one of the volume integrals of expression (15) into a flux integral through the dislocation surface $S_{\mathrm{d}}$ :

$$
\begin{aligned}
\delta I= & -\frac{e}{\tau} \int_{V_{\mathrm{d}}} \mathrm{e}^{-z / L}\left\{p_{0}(\mathbf{r})-p(\mathbf{r})\right\} \mathrm{d} \mathbf{r} \\
& +e D \int_{S_{\mathrm{d}}} \mathrm{e}^{-z / L} \operatorname{grad} \delta p(\mathbf{r}) \cdot \mathrm{d} \mathbf{S} \\
& +\int_{V_{\mathrm{d}}} \delta \mathbf{j}_{\mathrm{d}} \cdot \operatorname{grad}\left(\mathrm{e}^{-z / L}\right) \mathrm{d} \mathbf{r} .
\end{aligned}
$$

Similarly to what happens at the surface limiting the bulk material from the depleted region of a Schottky diode, one can consider that any minority carrier reaching the dislocation surface $S_{\mathrm{d}}$ is instantaneously drifted towards the dislocation line where it is recombined. Thus, within the volume $V_{\mathrm{d}}$ the terms $p(\mathbf{r})$ and $\delta \mathbf{j}_{\mathrm{d}}(\mathbf{r})$ cancel and the current variation is fully described by the simple expression :

$$
\begin{aligned}
\delta I \cong \frac{e}{\tau} \int_{V_{\mathrm{d}}} \mathrm{e}^{-z / L} p_{0}(\mathbf{r}) \mathrm{d} \mathbf{r}- \\
\quad-\mathrm{e} D \int_{S_{\mathrm{d}}} e^{-z / L} \operatorname{grad}(\delta p) \cdot \mathrm{d} S .
\end{aligned}
$$

The first integral is equivalent to that of Donolato's model [1] except that no defect strength has been introduced; it will be named $\delta I_{1}$ in the following. The second term, $\delta I_{2}$, arises because $p(r)$ has not been approximated to $p_{0}(\mathbf{r})$ as it has already be done [1]. As it will be shown by the numerical results, this term is predominant in the EBIC contrast formation; this stresses the need of a calculation of $p(\mathbf{r})$ which is the new distribution created in presence of the dislocation. In order to find it, the depleted region approximation around the dislocation line will be used (Read's model). It allows us to calculate the cylinder radius $R$, determined by the physical properties of the dislocation and of the host crystal. Each carrier reaching the dislocation cylinder is instantaneously recombined at the dislocation line. This may be simply depicted by assigning to the dislocation surface $S_{\mathrm{d}}$ an infinite recombination rate. Within this model, $p(\mathbf{r})$ determination can be simply made by solving equation (4) with an additional boundary condition : $p(\mathbf{r})$ must also vanish at any point of $S_{\mathrm{d}}$. 
2.1 DETERMINATION OF $p(r)$ IN PRESENCE OF A DISLOCATION PERPENDICULAR TO THE SURFACE. The determination of $p(r)$ cannot easily be done for any geometry of the incident electron beam versus the dislocation position. Looking only for the maximum contrast value and not for contrast profiles, we will restrict our calculation to the particular case where the electron beam impinges directly above the dislocation; so the configuration keeps a cylindrical symmetry. Since $\delta p(\mathbf{r})$ at the dislocation surface can be known from its expression outside the dislocation volume, the solution of the diffusion equation is only needed in the region where there is no electrical field.

$$
D \Delta p(\mathbf{r})=\frac{p(\mathbf{r})}{\tau}-g(\mathbf{r}) .
$$

As previously, let us write the new excess carrier density $p(\mathbf{r})$ as

$$
p(\mathbf{r})=p_{0}(\mathbf{r})+\delta p(\mathbf{r})
$$

$p_{0}(\mathbf{r})$ is solution of the diffusion equation without any defect ; then, outside the dislocation cylinder, $\delta p(\mathbf{r})$ is solution of :

$$
\Delta \delta p(\mathbf{r})=\frac{\delta p(\mathbf{r})}{L^{2}} .
$$

Because of the cylindrical symmetry, the variable separation method is used :

$$
\delta p(\mathbf{r})=\delta p(r, z)=F(r) . Z(z)
$$

$F$ and $Z$ satisfy the following differential equations :

$$
\begin{gathered}
\frac{\partial^{2} F}{\partial r^{2}}+\frac{1}{r} \frac{\partial F}{\partial r}+\left(\varepsilon^{2}-\frac{1}{L^{2}}\right) F=0 ; \\
\frac{\partial^{2} Z}{\partial z^{2}}=\varepsilon^{2} Z
\end{gathered}
$$

whose general solutions are :

$$
F(r)=J_{0}\left(\beta_{\varepsilon} r\right) \text { or } Y_{0}\left(\beta_{\varepsilon} r\right)
$$

with

$$
\beta_{\varepsilon}^{2}=\varepsilon^{2}-1 / L^{2}
$$

$J_{0}$ and $Y_{0}$ are the zero order Bessel functions of the first and second kind respectively, and

$$
Z(z)=\exp ( \pm \varepsilon z)
$$

$\varepsilon$ can be any kind of real or imaginary continuous variable. In order to fit the boundary conditions in a semi infinite medium, the solutions have to be a continuous linear combination of $F(r)$ and $Z(z)$ with respect to $\varepsilon$ :

$$
\begin{gathered}
\delta p(r, z)=\int\left[A(\varepsilon) J_{0}\left(\beta_{\varepsilon} r\right)+B(\varepsilon) Y_{0}\left(\beta_{\varepsilon} r\right)\right] \times \\
\quad \times[C(\varepsilon) \exp (\varepsilon z)+D(\varepsilon) \exp (-\varepsilon z)] \mathrm{d} \varepsilon .
\end{gathered}
$$

Let us notice that real values of $\varepsilon$ do not lead to solutions simultaneously vanishing at the surface $\Sigma$ of the Schottky diode and for infinite values of $z$. Thus $\varepsilon$ has to be an imaginary quantity : $\varepsilon=i \alpha$. It comes :

$$
\begin{array}{r}
\delta p(r, z)=\int\left[A(\alpha) K_{0}\left(\beta_{\alpha} r\right)+B(\alpha) I_{0}\left(\beta_{\alpha} r\right)\right] \times \\
\times[C(\alpha) \sin (\alpha z)+D(\alpha) \cos (\alpha z)] \mathrm{d} \alpha(28)
\end{array}
$$

with :

$$
\beta_{\alpha}^{2}=\alpha^{2}+1 / L^{2}
$$

where the variation range of $\alpha$ can be limited to positive values. $K_{0}$ and $I_{0}$ are now the zero order modified Bessel functions of the first and second kind.

In order to avoid divergency of $\delta p(r, z)$ for $r \rightarrow \infty, B(\alpha)$ has to be taken equal to zero. In order to obtain a solution vanishing at the collecting plane, $D(\alpha)$ has also to be taken equal to zero. Expression (29) becomes :

$\delta p(r, z)=\int_{0}^{\infty} K_{0}\left(\beta_{\alpha} r\right) \times C^{\prime}(\alpha) \sin (\alpha z) \mathrm{d} \alpha$.

Use of the boundary condition at the dislocation surface $(p(R, z)=0)$, allows the straightforward determination of the constants $C^{\prime}$ :

$$
\begin{aligned}
P_{0}(R, z)=-\int_{0}^{\infty} K_{0}\left(\beta_{\alpha} R\right) \times \\
\times C^{\prime}(\alpha) \sin (\alpha z) \mathrm{d} \alpha
\end{aligned}
$$

$R$ is the dislocation cylinder radius.

Let us multiply expression (31) by $\sin \left(\alpha^{\prime} z\right)$ and integrate along $z$ :

$$
\begin{array}{r}
\int_{0}^{\infty} p_{0}(R, z) \sin \left(\alpha^{\prime} z\right) \mathrm{d} z=-\int_{0}^{\infty} K_{0}\left(\beta_{\alpha} R\right) \times \\
\times C^{\prime}(\alpha) \sin (\alpha z) \cdot \sin \left(\alpha^{\prime} z\right) \mathrm{d} \alpha \mathrm{d} z .
\end{array}
$$

Using the identity obtained for positive values of $\alpha$ and $\alpha^{\prime}$ :

$$
\int_{0}^{\infty} \sin \left(\alpha^{\prime} z\right) \sin (\alpha z) \mathrm{d} z=\frac{\pi}{2} \delta\left(\alpha^{\prime}-\alpha\right)
$$

we obtain :

$$
C^{\prime}(\alpha)=-\frac{2}{\pi} \frac{\int_{0}^{\infty} p_{0}(R, z) \sin (\alpha z) \mathrm{d} z}{K_{0}\left(\beta_{\alpha} R\right)} .
$$

By replacing $p_{0}(R, z)$ by its expression given by (31), we obtain :

$$
\begin{aligned}
C^{\prime}(\alpha)=-\frac{2}{\pi} \int_{0}^{\infty} \int_{V} & \left.\chi\left(\mathbf{r}, \mathbf{r}^{\prime}\right)\right|_{r=R} \times \\
& \times \frac{g\left(\mathbf{r}^{\prime}\right) \sin (\alpha z)}{D K_{0}\left(\beta_{\alpha} R\right)} \mathrm{d} z \mathrm{~d} \mathbf{r}^{\prime} .
\end{aligned}
$$


Integration with respect to $z$ gives :

$$
\begin{aligned}
\int \chi\left(\mathbf{r}, \mathbf{r}^{\prime}\right) \sin (\alpha z) \mathrm{d} z= & \\
= & \frac{\sin \left(\alpha z^{\prime}\right)}{4 \pi} K_{0}\left(\beta_{\alpha}\left|\mathbf{r}-\mathbf{r}^{\prime}\right|\right)
\end{aligned}
$$

$\delta p(\mathbf{r})$ is finally given by :

$$
\begin{aligned}
\delta p(\mathbf{r})=- & \frac{1}{2 \pi^{2}} \int \frac{K_{0}\left(\beta_{\alpha} \mathbf{r}\right)}{K_{0}\left(\beta_{\alpha} R\right)} K_{0}\left(\beta_{\alpha}\left|\mathbf{r}-\mathbf{r}^{\prime}\right|\right) \times \\
& \times \frac{g\left(\mathbf{r}^{\prime}\right)}{D} \sin \left(\alpha z^{\prime}\right) \sin (\alpha z) \mathrm{d} \mathbf{r}^{\prime} \mathrm{d} \alpha .
\end{aligned}
$$

\subsection{EBIC CONTRAST OF A DISLOCATION.}

2.2.1 Calculation of $\delta I_{1}$. - The first term describing the variation of the collected current due to the recombination of the carriers within the dislocation cylinder corresponds to (expression (18)) :

$$
\delta I_{1}=e \int_{V_{\mathrm{d}}} \int_{V} g\left(\mathbf{r}^{\prime}\right) \chi\left(\mathbf{r}, \mathbf{r}^{\prime}\right) \mathrm{e}^{-z / L} \mathrm{~d} \mathbf{r} \mathrm{d} \mathbf{r}^{\prime}
$$

i.e., in cylindrical coordinates :

$$
\begin{aligned}
\delta I_{1}=\int_{0}^{2 \pi} \int_{0}^{R} & {\left[\int_{V} g\left(\mathbf{r}^{\prime}\right) \mathrm{d} \mathbf{r}^{\prime}\right] \times } \\
& \times r \mathrm{~d} r \mathrm{~d} \theta \int_{0}^{\infty} \chi\left(\mathbf{r}, \mathbf{r}^{\prime}\right) \mathrm{e}^{-z / L} \mathrm{~d} z .
\end{aligned}
$$

The last integral in (39) is found equal to :

$$
\begin{aligned}
& \int_{0}^{\infty} x\left(\mathbf{r}, \mathbf{r}^{\prime}\right) \mathrm{e}^{-z / L} \mathrm{~d} z= \\
& \quad .=\frac{1}{\pi^{2}} \int_{0}^{\infty} K_{0}\left(\lambda\left|\mathbf{r}-\mathbf{r}^{\prime}\right|\right) \frac{\sin \left(k_{z} z^{\prime}\right)}{\lambda^{2}} k_{z} \mathrm{~d} k_{z}(40)
\end{aligned}
$$

with $\lambda=\left(k_{z}^{2}+1 / L^{2}\right)^{1 / 2}$. Finally, $\delta I_{1}$ is given by :

$$
\begin{aligned}
\delta I_{1} & =\frac{2}{\pi L^{2}} \int_{0}^{R}\left[\int_{0}^{\infty} g\left(r^{\prime}, z^{\prime}\right) r^{\prime} \mathrm{d} r^{\prime} \mathrm{d} z^{\prime}\right] \times \\
& \times r \mathrm{~d} r \int_{0}^{\infty} \frac{K_{0}\left(\lambda\left|r-r^{\prime}\right|\right)}{\lambda^{2}} \sin \left(k_{z} z^{\prime}\right) k_{z} \mathrm{~d} k_{z} .
\end{aligned}
$$

2.2.2 Determination of $\delta I_{2}$. - Derivation of $\delta p(\mathbf{r})$ (Eq. (37)) with respect to $r$ gives for $\delta I_{2}$ :

$$
\begin{aligned}
\delta I_{2} & =\frac{e R}{\pi} \int \frac{g\left(\mathbf{r}^{\prime}\right)}{\beta_{\alpha}} \frac{K_{1}\left(\beta_{\alpha} R\right)}{K_{0}\left(\beta_{\alpha} R\right)} \times \\
& \times\left.\alpha \sin \left(\alpha z^{\prime}\right) K_{0}\left(\beta_{\alpha}\left|\mathbf{r}-\mathbf{r}^{\prime}\right|\right)\right|_{r=R} \mathrm{~d} \mathbf{r}^{\prime} \mathrm{d} \alpha
\end{aligned}
$$

$K_{1}$ is the first order modified Bessel function of the first kind. Expression (42) is numerically calculated.

\section{Results and discussion.}

Numerical results are listed in table I. They correspond to the case of a dislocation located in n-type GaAs $\left(n=4 \times 10^{16} \mathrm{~cm}^{-3}\right)$ covered with $10 \mathrm{~nm}$ of titanium to produce to Schottky barrier. The dislocation radius $R$ has been taken as the Read radius [12] :

$$
R=\left(\frac{n_{\mathrm{t}}}{n \pi p}\right)^{1 / 2}
$$

where $n_{\mathrm{t}} / \rho$ is the number of electrons trapped per dislocation unit length. A value of 0.5 for $n_{t} / \rho$ gives a radius $R=0.1 \mu \mathrm{m}$ (these values correspond to a dislocation one-dimensional band located at about $0.2 \mathrm{eV}$ above the valence band [13]).

The excess carrier generation function $g(\mathbf{r})$

Table I. - Calculated EBIC contrast of a dislocation perpendicular to the surface in $n$ type GaAs $\left(n=4 \times 10^{16} \mathrm{~cm}^{-3}\right)$ as a function of the bulk diffusion length $L$, of the accelerating voltage $E_{0}$ and of $R$, the dislocation radius.

\begin{tabular}{|c|c|c|c|c|c|}
\hline & $L(\mu \mathrm{m})$ & $E_{0}(\mathrm{kV})$ & contrast $(\%)$ & $\delta I_{1} / I_{\mathrm{bo}}(\%)$ & $\delta I_{2} / I_{\mathrm{bo}}(\%)$ \\
\hline \multirow{5}{*}{$R=100 \mathrm{~nm}$} & 0.3 & 30 & 0.73 & 0.09 & 0.64 \\
\cline { 2 - 6 } & 1 & 10 & 10 & 0.134 & 9.92 \\
& & 20 & 4.73 & 0.082 & 4.65 \\
\cline { 2 - 6 } & 10 & 30 & 2.33 & 0.048 & 2.28 \\
\hline \multirow{3}{*}{$R=50 \mathrm{~nm}$} & 1 & 30 & 3.9 & 0.001 & 3.9 \\
\hline
\end{tabular}


$\left(\mathrm{cm}^{-3} \mathrm{~s}^{-1}\right)$ chosen for the numerical application has been derived by Akamatsu et al. [14] from Monte
Carlo simulations; it depends on the accelerating voltage via the penetration depth $r_{1}$ :

$$
\begin{aligned}
g\left(r, z, r_{1}\right) & =a^{*}\left(1.1+7.13 \frac{z}{r_{1} / 3}\right)\left[\exp \left(-\frac{z^{2}}{2\left(r_{1} / 3\right)^{2}}\right) \cdot \exp \left(-\frac{r^{2}}{2\left(r_{1} / 3\right)^{2}}\right)+\right. \\
& \left.+3.4 \exp \left(-\frac{z^{2}}{2\left(r_{1} / 6\right)^{2}}\right) \cdot \exp \left(-\frac{r^{2}}{2\left(r_{1} / 9\right)^{2}}\right)+96 \exp \left(-\frac{z^{2}}{2\left(r_{1} / 12\right)^{2}}\right) \cdot \exp \left(-\frac{r^{2}}{2\left(r_{1} / 27\right)^{2}}\right)\right]
\end{aligned}
$$

$r_{1}$ is the Gruen range given in [15], and the prefactor « $\mathbf{a}$ » is such that :

$$
\int_{V} g\left(r, z, r_{1}\right) \mathrm{d} \mathbf{r}=g_{0}
$$

where $g_{0}$ is the total generation factor.

It has first to be noticed, as in Donolato's calculations [2], that the EBIC contrast decreases when the accelerating voltage $E_{0}$ increases. The contrast reaches few percents or few ten percents, which is of the same order of magnitude as the experimental ones. In table I are also reported the contribution of both terms to the EBIC contrast. The second term $\delta I_{2}$ is always the largest, even when the diffusion length is very small. Furthermore, it makes the contrast to increase with $L$; this would not have been the case if only the first term had been taken into account, in contrast with Donolato's results [2] where a defect strength (depending on $L$ ) is introduced.

The preeminent participation of the term $\delta I_{2}$ to the contrast implies that its magnitude is more sensitive to the accelerating voltage and to the diffusion length than to the dislocation cylinder radius.

The contrast has also been calculated for another generation function $g(\mathbf{r})$ given by [16] :

$$
g(\mathbf{r})=\frac{g_{0}}{r_{1}} \frac{\Lambda\left(z / r_{1}\right)}{2 \pi \sigma^{2}\left(z, r_{1}\right)} \exp \left(-\frac{r^{2}}{2 \sigma^{2}\left(z, r_{1}\right)}\right)
$$

$r_{1}$ is the Gruen range [15], $g_{0}$ is the total generation rate and $\sigma$ is the lateral Gaussian function width. It is taken as [16] :

$$
\sigma^{2}\left(z, r_{1}\right) \cong 0.11 z^{3} / r_{1}
$$

$\Lambda\left(z / r_{1}\right)$ is the depth-dose function derived by Everhart and Hoff [17] :

$$
\Lambda\left(z / r_{1}\right)=\left[\begin{array}{ll}
0.6+6.2\left(z / r_{1}\right)-12.4\left(z / r_{1}\right)^{2}+5.69\left(z / r_{1}\right)^{3} & 0 \leqq z / r_{1} \leqq 1.1 \\
0 & z / r_{1}>1.1
\end{array}\right.
$$

For diffusion lengths equal to one micron or greater, EBIC contrasts are a little larger than those obtained with the first generation function; for example, it is equal to $5.5 \%$ instead of $2.3 \%$ at $30 \mathrm{kV}$ for $L=1 \mu \mathrm{m}$, and the contribution of the second term is a little less than with the first function. This is due to the lateral extension of the generation function which is different in both cases. Let us notice that, when the diffusion length becomes very small $(L=0.3 \mu \mathrm{m})$, the contrast is very different from that calculated previously ( $12 \%$ instead of $0.73 \%$ ). However, the background EBIC current $I_{0}$ is three times smaller than that derived with the first generation function and does not correspond to experimental results [18]. This last remark invalidates therefore the contrast results. A possible reason for these discrepancies may stand in the fact, that the second generation function does not describe correctly the minority carrier distribution close to the surface in GaAs. This points out the fact that the choice of an analytical generation function $g(r)$ is crucial, not only if we want to derive the dislocation cylinder radius (and therefore the dislocation level position), but also if we want to describe the contrast dependence as a function of the crystal parameters (temperature, dopant concentration and diffusion length).

The numerical values listed in table I do not take into account any recombination at the dislocation in the space charge region. As it is not negligible, as shown in [21], its contribution to the total EBIC contrast will be much more important at low accelerating voltages, especially if the doping level of the semiconductor is low. This type of correction has also to be made at high accelerating voltages when the bulk participation to the EBIC current is not predominant; this can occur in short diffusion length materials. 


\section{Conclusion.}

Intrinsic properties of dislocations have been considered for the calculation of the EBIC contrast by introducing a self-consistent electric field within the diffusion equation. To the authors' knowledge, it is the first time that dislocation electric fields have been explicitly taken into account. The rigourous treatment proposed in the present paper does not allow to take into consideration saturations effects since they would introduce a $z$-dependent dislocation radius. However, approximate solutions to this problem are in progress. Thus, the present numerical results can formally be compared with experimental ones in the case of a minority carriers generation low enough to avoid such saturation effects.

Within this limit, the most remarkable fact of our calculation is that, without introducing any phenomenological parameter, the right order of magnitude of the EBIC contrast of dislocations has been simulated contrarily to previous theoretical treatments which only dealt with qualitative contrast behaviours. Let us notice that our contrast values associated with intrinsic dislocation properties are large enough to be experimentally observed; this is again opposite to the conclusions of several authors who claimed that intrinsic properties may only lead to too small values to be experimentally observed $[19,20]$. Finally, our theoretical results can be used to determine quantitatively the dislocation electronic levels from experiments made at an injection level as low as possible.

\section{Acknowledgements.}

The authors thank R. J. Tarento (LPM, CNRS Bellevue) for his comments on the manuscript.

\section{References}

[1] Donolato C., Optik 52 (1978/79) 19.

[2] Donolato C., Appl. Phys. Lett. 34 (1979) 80.

[3] PASEMANN L., Ultramicroscopy 6 (1981) 237.

[4] Pasemann L., J. Phys. Lett. France 45 (1984) L133.

[5] Jackubowicz A., J. Appl. Phys. 59 (1986) 2205.

[6] Ourmazd A., Cryst. Res. Technol. 16 (1981) 137.

[7] Wilshaw P. R. and Booker G. R., Microsc. Semicond. Mater. Conf., Oxford, 25-27 March 1985, Inst. Phys. Conf. Ser. 76 : section 8 (1985) 329.

[8] Donolato C., J. Phys. France 47 (1986) 171.

[9] Ourmazd A., Wilshaw P. R. and BOoKer G. R., J. Phys. Colloq. France 44 (1983) C4-289.

[10] Pasemann L., Phys. Status Solidi (a) 84 (1984) 133.

[11] MoRse P. M. and FeshBACH H., Methods of Theoretical Physics (McGraw-Hill Book Company) 1953.

[12] Read W. T., Philos. Mag. 45 (1954) 775.

[13] Farvacoue J. L., Vignaud D., Ferre D. and
DePraetere E., Materials Science Forum, Trans. Tech. Publ. 38-41 (1989) 1367.

[14] Akamatsu B., Henoc P. and Martins R. B., J. Microsc. Spectrosc. Electron. 14 (1989) 12a ;

HENOC P., private communication.

[15] Quantitative Scanning Electron Microscopy, Eds. D. B. Holt et al. (Academic Press) 1974, p. 57.

[16] Donolato C., Phys. Status Solidi (a) 65 (1981) 649.

[17] Everhart T. E. and Hoff P. H., J. Appl. Phys. 42 (1971) 5837.

[18] CaRTON P., private communication.

[19] Kittler M. and Seifert W., Phys. Status Solidi (a) 66 (1981) 573.

[20] Akamatsu B., Jean-Louis A. M., Papadopoulo A. C. and Henoc P., Phys. Status Solidi (a) 95 (1986) 557.

[21] Sieber B. and Philibert J., Philos. Mag. B 55 (1987) 575 ;

Sieber B., Philos. Mag. B 55 (1987) 585. 